\title{
An Indirect Measure of the Implicit Level of Presence in Virtual Environments
}

\author{
Steven Nunnally ${ }^{1}$ and Durell Bouchard ${ }^{2}$ \\ ${ }^{1}$ University of Pittsburgh School of Information Science \\ smn34@pitt. edu \\ ${ }^{2}$ Roanoke College Department of Math, Computer Science, and Physics \\ bouchardaroanoke.edu
}

\begin{abstract}
Virtual Environments (VEs) are a common occurrence for many computer users. Considering their spreading usage and speedy development it is ever more important to develop methods that capture and measure key aspects of a VE, like presence. One of the main problems with measuring the level of presence in VEs is that the users may not be consciously aware of its affect. This is a problem especially for direct measures that rely on questionnaires and only measure the perceived level of presence explicitly. In this paper we develop and validate an indirect measure for the implicit level of presence of users, based on the physical reaction of users to events in the VE. The addition of an implicit measure will enable us to evaluate and compare VEs more effectively, especially with regard to their main function as immersive environments. Our approach is practical, cost-effective and delivers reliable results.
\end{abstract}

Keywords: Virtual Environments, Presence, Indirect Implicit Measure.

\section{Introduction}

VEs are very important to many aspects as technology advances. Their immersive quality allows many different users to create situations that are either unavailable or impractical to create in real life. The uses of VEs range from simple spatial tasks of showing buildings to perspective buyers before the project is started to creating the same emotional and physical reactions in a high risk situation without the danger. Military, rescue, and medical personnel especially can utilize VEs to improve their skills without exposing themselves to risky situations while reducing cost at the same time, an important application since these personnel will soon be expected to act with precision in the worst of scenarios. Another great use of this emerging technology is fixing psychological disorders. VEs are being used to confront an individual's worst phobia or to create a reaction, to help treat disorders like Post-Traumatic Stress.

Unfortunately, this research is hindered because the different tools necessary to measure the different aspects of VEs are limited. The key to all of the applications listed above is the immersive quality that VEs possess. The term for this quality is presence, which is the user's perceived level of authenticity of the displayed 
environment. The above application must create the emotional and physical reactions to the presented situation in order to accomplish their goal of increasing efficiency when the real situation occurs [5]. Direct measures, like questionnaires, have been used in the past to measure the direct effect of the environment on the user. Researchers have worked to validate numerous questionnaires that not only support evidence that one VE has a higher level of presence than another, but can often give evidence to determine the specific trait that increases the level of presence [6]. These direct measures help researchers compare different features of different VEs to find which enables the greatest direct increase of presence on the user. However, some features may not consciously affect the user, making a direct measure insufficient.

To advance research in this field, we must also be able to determine the indirect effects that the VEs have on users by measuring the implicit levels of presence as well as the explicit levels. In related work in Psychology such implicit measures have proven to be better predictors of behavior than direct and explicit measures, which could prove more important in an application where the emotional and physical reaction is the key to success [4]. Currently, no indirect measurement of the implicit level of presence is readily available for researchers.

There are a few earlier studies which have attempted to find an indirect measure of the implicit level of presence. One of these studies attempted to use a method they named behavioral realism, which measures a reaction to an event within the environment. One attempt was with postural responses [2]. Freeman et al. tried this using a video of a car racing around a race track while measuring the subject's response to the hairpin turns. They used a questionnaire in order to compare the sway data with the questionnaire data. They did this using many different presence altering features. One was stereoscopic vs. monoscopic video and another was different screen sizes $[1,2]$. They concluded that their data showed weak support for the use of this behavioral realism measurement in evaluating the VE features. The indirect measure did not correlate with the questionnaire.

The conclusion rejected the hypothesis because it was necessary for the direct measure and indirect measure to correlate, which would only be coincidental if there was correlation because the measurements simply measure presence in different ways [3]. First there was not a large enough difference between the features that were used to increase presence given the number of participants. Tan showed that participants performed tasks much better on larger displays than on smaller ones, even when the visual angle was the same, supporting the idea that different field of views (FOVs) should affect presence [5].

The experiment detailed in this paper uses the CAVE at Roanoke College to rework the screen size experiment of Freeman. This CAVE encapsulates 170 degrees of the user's FOV for the larger display, as compared to Freeman's 50 degrees. Freeman also used a passive activity in the attempt to measure presence. The level at which the user is involved in the VE can also greatly affect presence [6]. This measurement uses an active response to measure presence and might only show results with an active environment. Finally, the hypothesis more correctly states that this same measurement can be used to measure presence because of the higher level of involvement and the greater difference between the features of the VEs. This will be supported by showing that this measurement rejects the hypothesis that the CAVE, or immersive condition, and the desktop display, or non-immersive condition, has a 
lesser or equal level of presence. Further, the measurement will show a greater reliability than the explicit measure of presence, with more consistent results and a greater confidence.

\section{Experimental Evaluation}

\subsection{Procedure}

The design listed here is meant to correct the problems in the experiment described above. This experiment is a 1 by 1 comparison, for a more direct and less complicated experiment. Every subject was administered the test on both the non-immersive and the immersive condition to set up a within subject comparison, half started with the non-immersive condition.

Participants actively navigated a virtual racetrack using a steering wheel and foot pedals to drive the racecar. The steering wheel was set up so that it could not adjust so the device would not accidentally move during the test, creating false movement in the postural sway. Participants were allowed to adjust their chair at the beginning of the experiment for comfort, but were then asked to keep the chair's position fixed so that the data would be taken from the same distance and the FOV would be comparable between conditions.

Participants wore suspenders and a head band that had infrared lights attached. With these lights, a Wiimote was used to track the person's head and shoulder position to determine their sway. The character's physical location and direction in the virtual environment was logged about every 30 milliseconds as was the position of the 4 infrared lights. The participants were not told about the indirect measure of the implicit level of presence, to not bias the results.

The participant was then given a calibration sequence. First the steering wheel was turned in both directions with as little head and body movement as possible. Then the user turned both the steering wheel and their head simultaneously in both directions. This sequence was later used to minimize the effects of head turning and shouldering moving used to complete the task so that only postural sway based on a higher implicit level of presence was measured, instead of a difference between the screen sizes, as the user will be more likely to turn their head for the immersive condition.

The racetrack had many different types of curves and turns to force the participant to take turns at different speeds. The participants were allowed a short training period, which was the same course in the opposite direction. The subject could get used to the environment and the control aspects of the experiment during this training period. At this time the participant would fill out the questionnaire to get used to the questions, so knowledge of the questionnaire did not bias the results of the second direct measurement. The course was a circuit, so that the participants would not finish before the trial time expired. The participants were asked to stay on the track and warned that bumps and hills off the track were designed to slow them down.

After each trial the subjects were given a presence questionnaire to get the direct measure of presence of the condition (shown in Fig. 1). There were four questions. The first three were used to directly measure their explicit level of presence, while the fourth was used to determine whether or not the subject should continue with the experiment. 
1. To what extent did you feel present during the test - as though you were "really there"?

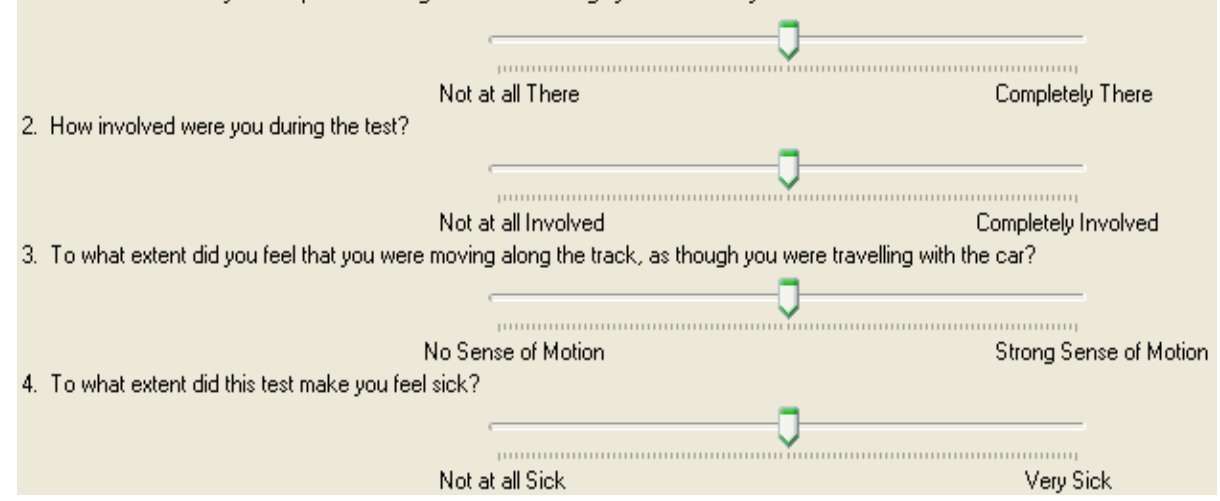

Fig. 1. The questionnaire used for this experiment

Twenty-two Roanoke College undergraduate students, eighteen male, volunteered for this experiment. A $\$ 25 \$$ prize was awarded to the participant with the fastest lap time as an incentive used to increase involvement, adding to the presence of both VEs in the experiment.

\subsection{Apparatus}

Most of the simulation is handled by Epic Games' Unreal Tournament Game Engine (2003). Epic Games created this engine for a first-person shooter video game, but the weapons and crosshairs were made invisible for the purposes of this experiment. The racetrack was developed using Epic Games' Unreal Editor. The movements were controlled using Gamebots, which is a command based language that passes messages between the user and the game character. This allows for a program to control the character's movement so that the character moves like a car, with acceleration and deceleration, depending on the pedals position. This could also log the input device variables for analysis.

The input device was a Logitech steering wheel and foot pedals. A program calculated the speed and rotational direction based off of the previous state of the character and the current state of the input device. Just like a normal car, the accelerator pedal would accelerate the car faster the further the pedal was pushed. The car would decelerate if no pedals were pushed and decelerate faster if the brake was pushed.

The participants' motion was recorded using 2 Wii sensor bars that are infrared lights mentioned in the previous section. They were attached to the participant with Velcro, using suspenders to trace the shoulders and a head band to trace the head (shown in Fig. 2). The lights were recorded with a Wiimote, which passed the information in pixel coordinates to a program which recorded the coordinates of the 4 infrared lights. These coordinates were used to measure the subject's position. 


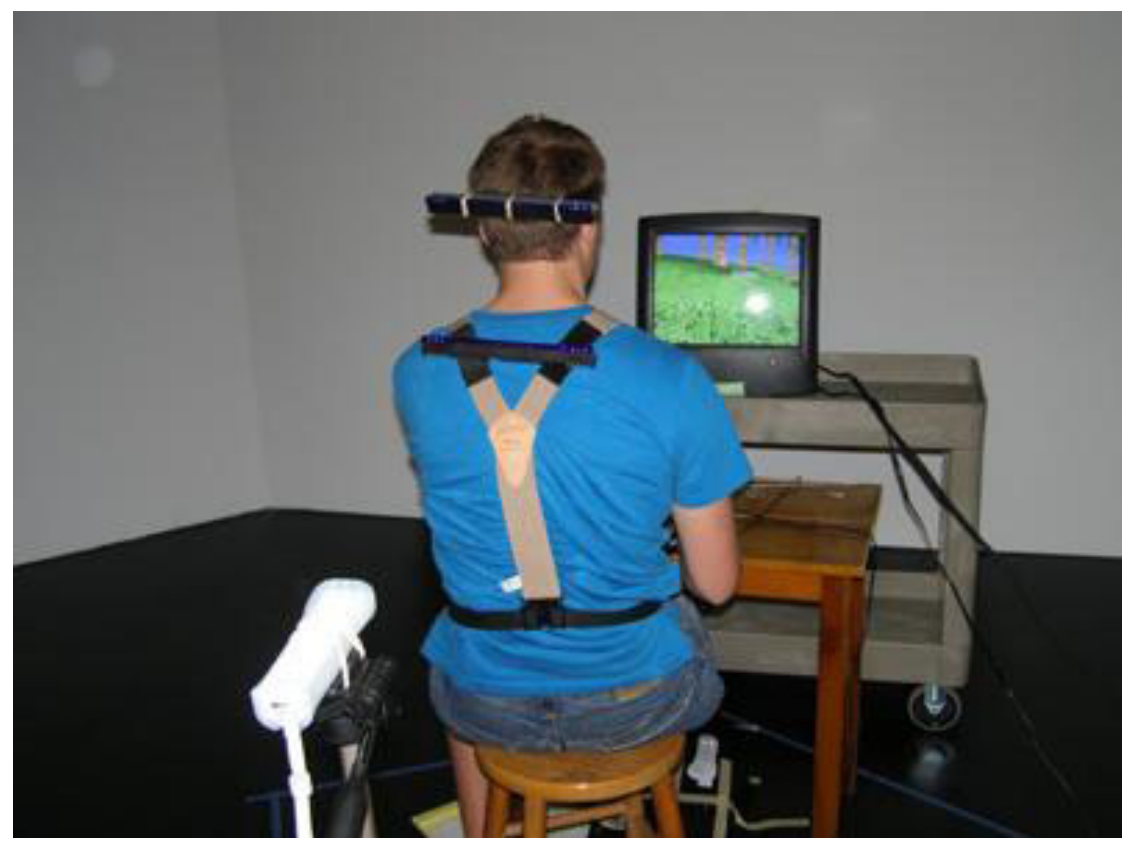

Fig. 2. Apparatus used to measure the users body position. The user is also using the steering wheel and foot pedals and the screen used for the non-immersive condition.

The display for the small screens was on a standard 18 inch CRT display, which fills a FOV of about 28 degrees. The large screen was displayed on the CAVE, which fills a FOV of about 170 degrees. The CAVE uses 4 Epson Projectors to project the image on three wall sized screens. The middle screen is $12 \mathrm{ft}$. wide by $8 \mathrm{ft}$. tall and the other two screens act as wings that are both $6 \mathrm{ft}$. wide by $8 \mathrm{ft}$. tall. The wings wrap around the user slightly to gain the 170 degrees FOV advantage.

\section{Analysis}

The analysis for the indirect measure begins with two sets of data: the character's rotational position and the four infrared light coordinates. Both have synchronized timestamps for all values. First, the measurement must be derived from the raw data. The recorded character's rotational position is not useful for this measurement, but the difference between the rotational values represents the position of the steering wheel, which is used to determine when the event of turning the vehicle occurs and to what extent. Next, some interpolation must be used for the data. The infrared lights were recorded as a pixel coordinate, but whenever one pixel was not recorded the $\mathrm{x}$ and $\mathrm{y}$ position of that light was recorded as a zero. All of these zeros were replaced using interpolation from the first point before the light was dropped to the next available point. Next, the timestamps of both must match to allow direct comparison between the two sets of data. The steering wheel position started and ended with each trial, and was therefore the base data that was used for comparison. The infrared coordinates 
were thus taken as a weighted average for each of the timestamps used alongside the steering wheel position. This gives an interpolated position of the subject's position for each record of the steering wheel position.

The steering wheel data can range to some point number $\mathrm{n}$ in both the negative and positive direction so that the center position of the steering wheel is zero. The subjects' physical position must match this condition in order to compare the results, so that they are comparable. This method takes the average horizontal position, or $\mathrm{x}$ value, of all four infrared lights and averages them any time the steering wheel data are within the $10 \%$ range in the center of its minimum and maximum number to find the subject's resting location. It is assumed that if the steering wheel is near the center, than the vehicle is traveling in a near straight direction and no centripetal acceleration would be felt by the driver. Now each value of the averaged horizontal position is changed so that it represents the difference from the resting point, and therefore ranges from some number negative number to some positive number and the resting position is near zero, much like the steering wheel data. The data are then normalized so that both sets range from -1 to 1 using the minimum and maximum points of both sets.

\section{Subject 12 Big Data}

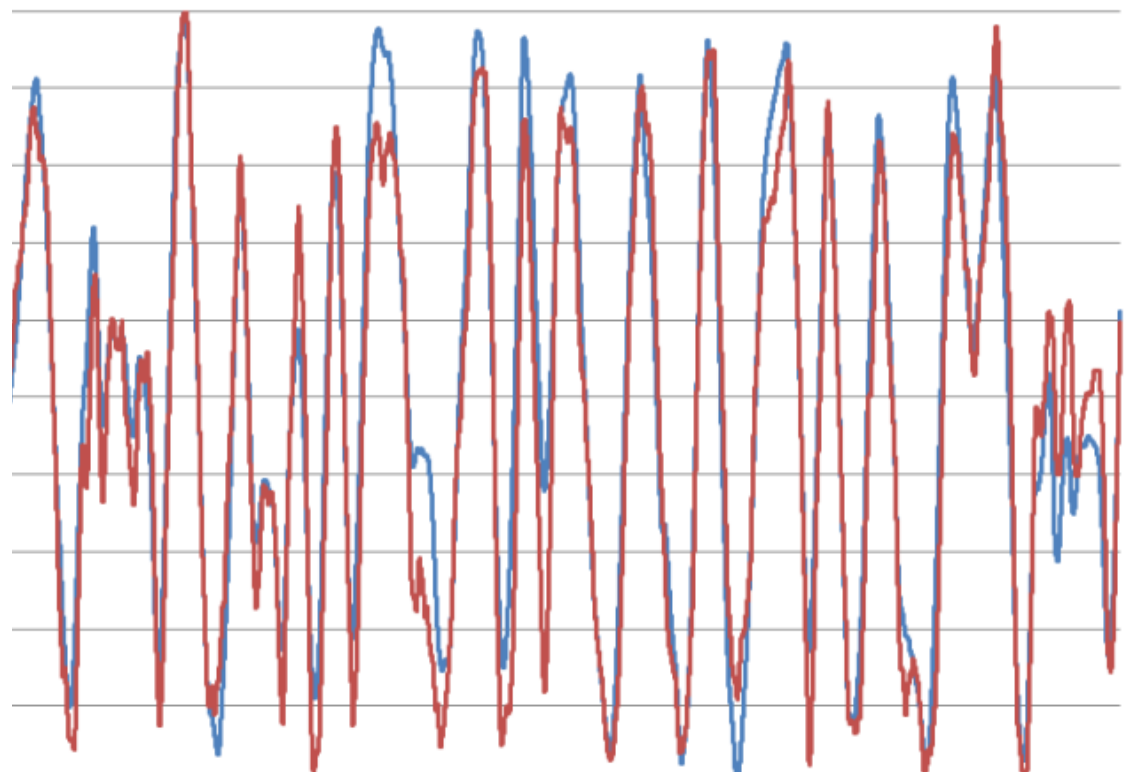

Fig. 3. A graphical representation of the data before the correlation is calculated. The lines represent the steering wheel position and the participants position away from their resting point.

Then, the calibration taken during the experiment was used to take away all motion not related to postural sway. The non-immersive condition used only the steering wheel calibration, since the screen is not large enough for head turning. The immersive condition used the other calibration sequence described in the procedure. The 
calibration data was used taking the subject's horizontal position change based on the percentage the steering wheel is turned. This value is added to the horizontal position at all times based on the amount the wheel is turned and which condition is in trial. To find the percentage of correlation (shown in Fig. 3) between the two sets of data, the number with the least absolute value is divided by the number with the greatest absolute value at each timestamp. This value is then averaged through the trial to find the presence value of that condition. This number is between -1 and 1 , where -1 shows completely negative correlation, an unlikely possibility with this measurement, zero shows no correlation, and thus no implicit level of presence, and 1 shows a completely positive correlation, and thus a perfect implicit level of presence is felt.

The questionnaire data was simple to analyze. The first three answers are a value between 0 and 100. These values are averaged since the questions ask about presence in different ways. This should minimize deviation with a greater number of answers for better accuracy in the VE's presence value. A greater number should show greater presence.

\section{Results}

The immersive conditioned produced a 0.1903 average correlation, whereas the nonimmersive condition only had a 0.0338 average (as shown in Fig. 4). 21 of the 24 participants' values confirmed that the CAVE is more immersive, with a presence value greater for the immersive condition. The difference between the values for the conditions was significant for this measurement $(\mathrm{p}<0.00001)$.

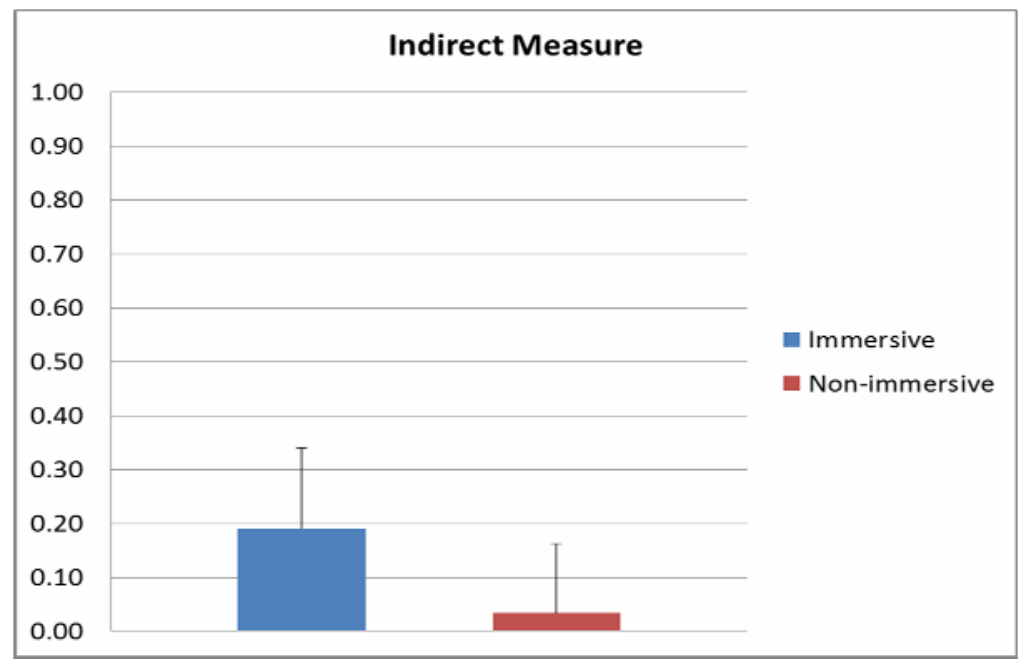

Fig. 4. A graph of the results for the indirect measure of the implicit level of presence

The direct measure of the explicit level of presence had an average value of 66.5909 for the immersive condition and 58.7121 for the non-immersive condition (as 
shown in Fig. 5). Only, 15 of the 24 participants' values confirmed that the CAVE is more immersive, but the difference was significant for this measurement $(\mathrm{p}<0.03)$.

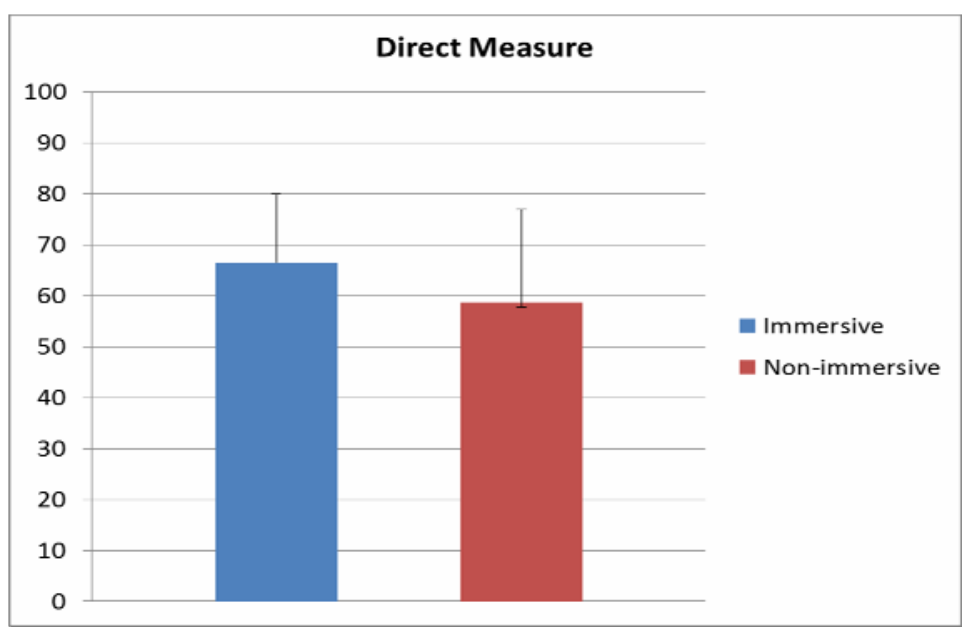

Fig. 5. A graph of the results for the direct measure of the explicit level of presence

\section{Conclusions}

The experiment produced evidence supporting the use of the indirect measure of the implicit level of presence for research with VEs. Furthermore, it does so more reliably and with a higher confidence level than the direct measure of the explicit level or presence used in this study. This shows that the direct measurement of the explicit level of presence fails to capture important aspects of a VE that relate to subconscious processes which can have great impact on behavior. This adds a powerful tool to VE researchers' arsenal in order to advance the technology. The utility offered by this new measurement offers great insight into the differences of implicit and explicit levels of presence using an indirect and direct level of presence.

\section{Future Work}

Our results motivate further work in which our measure can be used to predict the behavior of participants, particularly for events that aim to induce emotional response or reflexes. The validated measurement should be tested against different questionnaires to see if this is comparable to other questionnaires, and discover the advantage of using one over another. This experiment used a very simple questionnaire from Freeman et al.'s experiments, but more complex questionnaires exist.

Making this research more usable with different behavioral events should also be considered and tested. Our measurement only works with tasks that can make use of centripetal acceleration, like the driving task presented here. This is limiting because 
it may not be suitable for all researchers to test their presence enhancing features in a driving VE. This is an example of how this specific indirect measurement can work. For other tasks, possibly more generic tasks, one should seek new implicit measures. Also, the method of analyzing the data uses the minimums and maximums within the data sets to normalize the data which could minimize bias between subjects if the bias between users' and their movement comes from predetermined movement extremes. Additional Experiments could confirm or deny this idea, which could aid the research efforts by creating easier comparisons between experiments.

If these steps are achieved then indirect measures of presence can be used alongside direct measures to discover differences in VEs that have not yet been possible. Testing could begin to decide whether or not different senses like sounds or smells could have a significant effect on presence. A cost-benefit analysis could be completed so each feature of a VE can be examined for its price and the added presence value that is achieved. This could help schools and companies who may currently have doubts by showing exactly what presence value they will obtain for a certain price tag. Then schools, medical facilities, or the military can consider whether or not it is affordable to introduce as a new training or exploratory environment to aide in these efforts.

Acknowledgments. At Roanoke College I would like to thank Dr. Bouchard and Dr. Childers for helping me with the project. At the University of Pittsburgh I would like to thank Dr. Lewis and Dr. Kolling for supporting the project. Special thanks to Dr. Hughes for starting my interest in this project.

\section{References}

1. Freeman, J., Avons, S.E., et al.: Effect of Stereoscopic Presentation, Image Motion, and Screen Size on Subjective and Objective Corroborative Measures of Presence. Presence 10(3), 298-311 (2001)

2. Freeman, J., Avons, S.E., et al.: Using Behavioural Realism to Estimate Presence: A Study of the Utility of Postural Responses to Motion-Stimuli. Presence 9(2), 149-164 (2000)

3. Hofmann, W., Gawronski, B., Gschwendner, T., Le, H., Schmitt, M.: A Meta-Analysis on the Correlations Between the Implicit Association Test and Explicit Self-Report Measures. University of Trier, Germany (2004) (unpublished manuscript)

4. De Houwer, J.: What Are Implicit Measures and Why Are We Using Them. In: The Handbook of Implicit Cognition and Addiction, pp. 11-28. Sage Publishers, Thousand Oaks (2006)

5. Tan, D.S., Gergle, D., et al.: Physically Large Displays Improve Performance on Spatial Tasks. ACM Transactions on Computer-Human Interaction 13(1), 71-99 (2006)

6. Witmer, R.G., Singer, M.J.: Measuring Presence in Virtual Environments: A Presence Questionnaire. Presence 7(3), 225-240 (1998) 